\section{Does the Evidence Support the SHEA-IDSA Recommendation on the Use of Positive- Pressure Mechanical Valves?}

To the Editor-Few, if any, issues related to bloodstream infections and invasive devices have inspired such divergent opinions as that of needleless access connectors (ie, mechanical valves [MVs]). In a situation in which product choice is often decided on the basis of recommendations of societies such as the Society for Healthcare Epidemiology of America (SHEA) and the Infectious Diseases Society of America (IDSA), the obligation to ensure that such recommendations find support in empirical studies is paramount.

In the article titled "Strategies to Prevent Central LineAssociated Bloodstream Infections in Acute Care Hospitals" by Marschall et al. ${ }^{1}$ that was recently published in this journal, the following "recommendation" (section 4, subsection III, point 3) is made:

3. Do not routinely use positive-pressure needleless connectors with mechanical valves before a thorough assessment of risks, benefits, and education regarding proper use (B-11)..$^{88-91}$

a. Routine use of the currently marketed devices that are associated with an increased risk of CLABSI [central line-associated bloodstream infection] is not recommended. ${ }^{1(\mathrm{ps} 26)}$

The part of this recommendation that reads "before a thorough assessment of risks, benefits, and education regarding proper use" is a broad statement that should be in practice at all patient care facilities that have implemented the use of any new medical device. However, if read in conjunction with the rest of this recommendation, then a reasonable interpretation can lead to the conclusion that use of positive-pressure MVs are associated with an increased rate of CLABSI and, therefore, are not recommended for use. The authors' recommendations are overly broad and not very inclusive in suggesting that (1) the use of all positive-pressure MVs lead to increased risk of CLABSI and (2) the use of only positivepressure MVs are associated with this risk.

Indeed, only 2 (ie, Maragakis et al. ${ }^{2}$ and Rupp et al. ${ }^{5}$ ) of

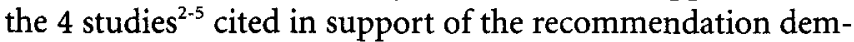
onstrated an increased rate of CLABSI when only a positivepressure MV was used. Moreover, in both of those studies, ${ }^{2,5}$ the same brand of positive-pressure MV was implicated. Second, the evidence used to support the recommendation is limited and lacks the scientific rigor necessary to make such a strong recommendation.

The recommendation lists only positive-pressure MVs and omits negative-pressure MVs, even though 2 (ie, Field et al. ${ }^{3}$ and Salgado et al. ${ }^{4}$ ) of the 4 studies ${ }^{2-5}$ cited to make the rec- ommendation used negative-pressure devices (Figure). Thus, the recommendation summarized the evidence incorrectly.

Each of the 4 studies identifies deficits related to the design of the MV that may be associated with an increase in the rate of catheter-related bloodstream infection (CRBSI). The following quotations from each of the 4 studies describes these specific design deficits:

MV devices have intricate access surfaces that are more difficult to disinfect than simpler split-septum models. The fluid path in the MV devices has moving parts, and at least 1 of the MV devices has internal corrugations that may serve as reservoirs and foster the growth of microbial contaminates....Some of the devices have been noted by healthcare personnel to have incomplete flushing of blood from the fluid channel, and some are opaque, so that this would not be readily apparent to the user. ${ }^{2(p 69)}$

We speculate that risk of colonization of the connector device may be higher for MV devices because of the potential difficulty in sterilizing the gap between the valve and the hub. ${ }^{3 /(p 612-613)}$

$[0]$ ur findings, along with the findings of other investigators, suggest that the mechanical valve system could be more difficult to disinfect because of the complicated nature of the multipart device. $^{4(p 687)}$

Upon close inspection of the valve (figure 2), one can observe a shallow depression and a rim between the diaphragm and the plastic housing. It is possible that microbes and debris could

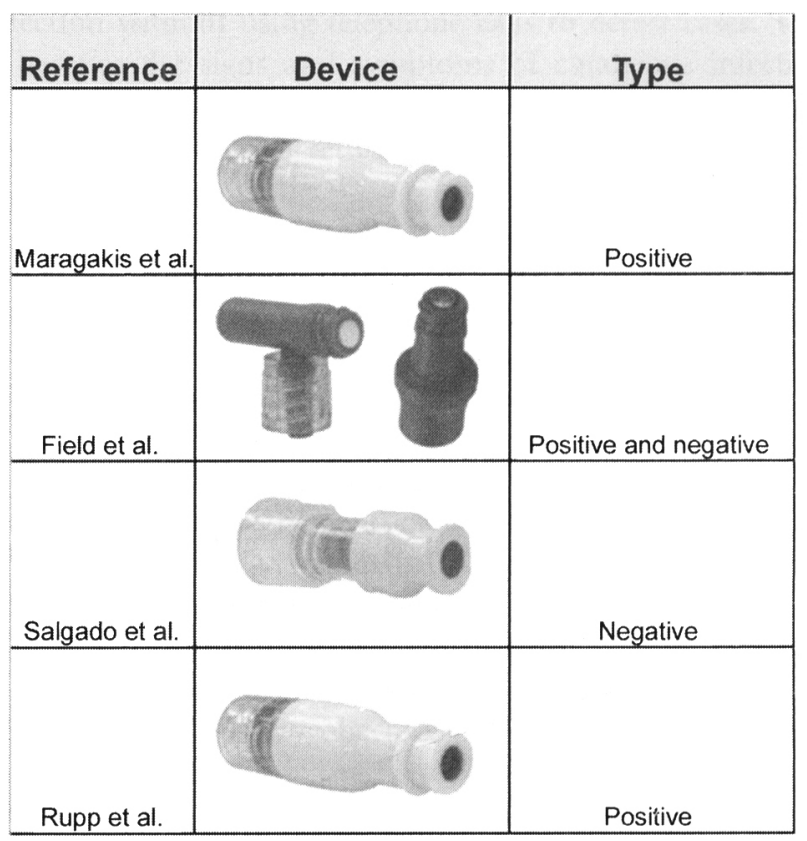

FIGURE. The positive- and negative-pressure devices associated with an increase in the rate of catheter-related bloodstream infection, according to the 4 studies cited. ${ }^{2-5}$ 
collect in this area, which would be relatively resistant to cleansing or disinfection. The internal mechanism of the valve contains moving parts which introduces irregularities in the fluid flow and may promote areas of stagnation and create potential reservoirs for microbial growth. Also, the plastic housing is opaque, which prohibits visual inspection of the connector valve. Therefore, it is possible that blood or infusion products could collect within the valve and, because of its opaque nature, go unnoticed by heal thcare workers. ${ }^{5(\mathrm{p} 1412)}$

In addition, the evidence used to support the recommendation is limited and lacked the proper control for variables that possibly influenced the results. The following limitations apply to all 4 studies:

1. The data were based on observation and were collected without randomization or proper control for variables.

2. The data were "retrospective, observational, and uncontrolled"5(p1412) and were collected during different periods of time, with likely differences in staff, patient populations, and level of care.

3. Each of the 4 studies reports on observational data from only a single healthcare facility. ${ }^{2-5}$

4. No data were presented that were related to the homogeneity of the patient population, assuming that the patient populations were the same, because they could have been, and very well may have been, dramatically different.

5. No specific data were presented that were related to the homogeneity of the specific catheter types used, the length of catheterization, or the insertion and maintenance techniques used.

6. The MVs studied were not utilized according to the manufacturer's instructions for use.

Finally, the recommendation fails to cite 2 studies $^{67}$ that demonstrate positive outcomes associated with the use of a positive-pressure connector. In the first study, by Garcia et al., ${ }^{6}$ the use of a positive-pressure connector was compared with the use of a split-septum device (ie, the split-septum device associated with lower CRBSI rates in Field et al., ${ }^{3} \mathrm{Sal}$ gado et al., ${ }^{4}$ and Rupp et al. ${ }^{5}$ ) for its impact on BSI rates in a 427-bed tertiary care hospital. Garcia et al. ${ }^{6}$ found that, at $95 \%$ confidence intervals, the $P$ values did not indicate a statistically significant difference in the BSI rates between the split-septum device group and the Luer activated device group of patients with peripheral and central lines. However, the Luer activated device group was associated with a lower occurrence of sharps injuries related to intravenous port access. The second study, by Costello et al., ${ }^{7}$ reported on a systematic intervention to reduce CLABSI rates in a pediatric cardiac intensive care unit from 7.8 to 2.3 cases of CLABSI per 1,000 catheter-days. Costello et al. ${ }^{7}$ reported:

For access to the CVLs [central venous lines], we converted our needleless connector system from a Luer lock-activated valve system...to a device that has a flat access surface and contains a positive-displacement valve....The positive-displacement valve has a fully cleanable surface and eliminates retrograde flow into the catheter when an infusion device is disconnected from an infusion port. $^{7\left(\text { (p18) }^{918}\right.}$

A compendium is a summary or abstract containing the essential information in brief form. This portion of this compendium left out essential information by omitting negativepressure MVs and the specific, well-documented deficits of MVs known to increase the risk of BSI. Because of the failure to include this relevant information and because of the lack of scientific rigor in the studies cited, we are asking that this recommendation be removed from the compendium. A critical assessment of all of the available literature on the efficacy of needleless access devices are needed; until then, recommendations related to the use of these devices should be limited to suggesting a thorough assessment of risks, benefits, and education regarding proper use of all devices in this category.

\section{Kerry J. Edgar, BS}

From Marketing and Clinical Affairs, Medegen, Ontario, California.

Address reprint requests to Kerry Edgar, BS, Marketing and Clinical Affairs, Medegen, 930 Wanamaker Avenue, Ontario, CA 91761 (kerry.edgar@ medegen.com).

Infect Control Hosp Epidemiol 2009; 30:402-403

(C) 2009 by The Society for Healthcare Epidemiology of America. All rights reserved. 0899-823X/2009/3004-0019\$15.00. DOI: $10.1086 / 596729$

\section{REFERENCES}

1. Marschall I, Mermel LA, Classen D, et al. Strategies to prevent central line-associated bloodstream infections in acute care hospitals. Infect Control Hosp Epidemiol 2008; 29:S22-S30.

2. Maragakis LL, Bradley KL, Song $X$, et al. Increased catheter-related bloodstream infection rates after the introduction of a new mechanical valves intravenous access port. Infect Control Hosp Epidemiol 2006; 27:67-70.

3. Field $\mathrm{K}$, McFarlane $\mathrm{C}$, Cheng $\mathrm{AC}$, et al. Incidence of catheter-related bloodstream infection among patients with a needleless, mechanical valvebased intravenous connector in an Australian hematology-oncology unit. Infect Control Hosp Epidemiol 2007; 28:610-613.

4. Salgado CD, Chinnes L, Paczesny TH, Cantey JR. Increased rate of catheter-related bloodstream infection associated with the use of a needleless mechanical valve device at a long-term acute care hospital. Infect Control Hosp Epidemiol 2007; 28:684-688.

5. Rupp ME, Sholtz LA, Jourdan DR, et al. Outbreak of bloodstream infection temporally associated with the use of an intravascular needleless valve. Clin Infect Dis 2007; 44:1408-1414.

6. Garcia R, Jenderesky L. A study of the effects on bacteremia and sharps injury rates after introduction of an advanced luer activated device (LAD) for intravascular access in a large hospital setting. Am J Infect Control 2007; 35:E75.

7. Costello JM, Morrow DF, Graham DA, Potter-Bynoe G, Sandora TJ, Laussen PC. Systematic intervention to reduce central line-associated bloodstream infection rates in a pediatric cardiac intensive care unit. Pediatrics 2008; 121:915-923.

\section{Reply to Edgar}

The journal recently printed our supplement article: "Strategies to Prevent Central Line-Associated Bloodstream Infec- 